\title{
Preface: semantic and conceptual issues in geographic information systems
}

\author{
Esteban Zimányi
}

Received: 27 October 2009

Accepted: 12 November 2009/Published online: 9 December 2009

(C) Springer Science+Business Media, LLC 2009

Recent advances in information technologies have increased the production, collection, and diffusion of geographical data, thus favoring the design and development of geographic information systems (GIS). Nowadays, GIS are emerging as a common information infrastructure that penetrate into more and more aspects of our society. This has given rise to new methodological and data engineering challenges in order to accommodate new users' requirements for new applications. Conceptual and semantic modeling are ideal candidates to contribute to the development of the next generation of GIS solutions. They allow to elicit and capture user requirements as well as the semantics of a wide domain of applications. The series of International Workshops on Semantic and Conceptual Issues in Geographic Information Systems (SeCoGIS) is intended to bring together researchers, developers, users, and practitioners carrying out research and development in geographic information systems. The selected papers of this special issue are extended versions of papers presented at the $3^{\text {rd }}$ International SeCoGIS workshop held in Barcelona in November 2008, in conjunction with the $27^{\text {th }}$ Conceptual Modeling Conference (ER).

The first paper of this special issue is entitled "Managing sensor traffic data and forecasting unusual behavior propagation" and is co-authored by C. B. Medeiros, M. Joliveau, G. Jomier, and F. De Vuyst. The authors introduce a complete framework for the integration of sensor data in the context of intelligent transportation systems, an emerging challenging area for applications of real-time GIS. The paper develops a data integration strategy for summarizing and filtering incoming sensor-based data. The second component of the contribution develops several querying and analysis strategies at different levels of granularity in space and time, thus facilitating the derivation of unusual patterns and behaviors. The whole approach has the advantage of being tested in real contexts.

The second paper, entitled "Exploiting geographic references of documents in a geographical information retrieval system using an ontology-based index" and written by N. R. Brisaboa, M. R. Luaces, A. S. Places, and D. Seco, presents a geographical dataretrieval approach that relies on an ontology-based index structure. The index structure combines spatial, textual, and ontology criteria. The advantage of this approach is that the semantics contained within an ontological representation of a given geographical database can facilitate retrieval procedures when searching for some items of semantic and

E. Zimányi $(\bowtie)$

Department of Computer and Decision Engineering (CoDE), Université Libre de Bruxelles,

50 av. F.D. Roosevelt, 1050 Bruxelles, Belgium

e-mail: ezimanyi@ulb.ac.be 
geographical interest. The approach is based on the OWL language, is supported by an experimental architecture, and preliminary performance evaluations are given.

The third paper, "A semantic and language-based representation of an environmental scene", is co-authored by J. M. le Yaouanc, E. Saux, and C. Claramunt. The authors present a cognitive and qualitative approach for the integration and description of environmental scenes. A language-based representation of a given environmental scene using panoramic photographs is applied, which facilitates derivation of the terms and constructs used in such descriptions. Several complementary schematic views of such linguistic descriptions are derived: either linguistics, semantics, or spatial. These complementary representations provide a tentative conceptualization of the cognitive abstractions applied in scene descriptions.

The fourth paper, entitled "OCL for formal modeling of topological constraints involving regions with broad boundaries", is co-authored by L. Bejaoui, F. Pinet, M. Schneider, Y. Bédard, and L. Bejaoui. This work introduces a modeling approach for the representation of topological constraints on top of the Object Constraint Language (OCL). It takes into account objects with vague shapes and broad boundaries, and adverbial constructs that refine the modeling of these topological relationships. The approach is implemented and experimented in the context of agricultural data.

The last paper of this special issue, "A knowledge infrastructure for intelligent query answering in location-based services" is written by S. Yu and S. Spaccapietra. The authors introduce a preliminary knowledge infrastructure for the development of location-based queries. The paper provides a discussion that addresses the peculiarities of location-based queries when compared to usual geographical queries, the potential contribution of a modular ontology, and the principles of development for application-independent approaches.

As shown by the large panorama of topics addressed by this selection of papers, the development of semantic and conceptual GIS potentially cover a large range of connected research domains. I am grateful to the authors and referees for their effort in selecting and reviewing the papers of this special issue. Special thanks to Andrew Frank for his efforts in the final reviewing process.

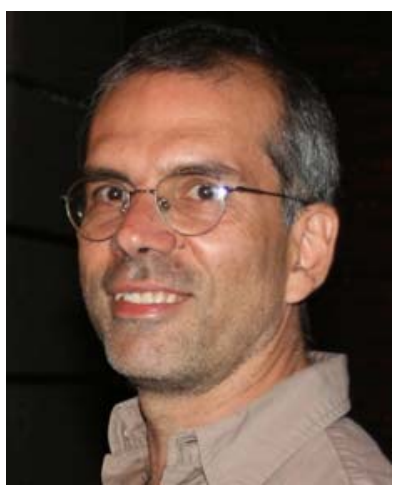

Esteban Zimányi is a professor and director of the Department of Computer and Decision Engineering (CoDE) of the Université Libre de Bruxelles (ULB), Belgium. He started his studies at the Universidad Autónoma de Centro América, Costa Rica. He received a B.Sc. (1988) and a doctorate (1992) in computer science from the Faculty of Sciences at the ULB. During 1997, he was a visiting researcher at the Database Laboratory of the Ecole Polytechnique Fédérale de Lausanne, Switzerland. His current research interests include semantic web and web services, geographic information systems, spatio-temporal databases, and data warehouses. He has co-authored and co-edited four books and published more than 80 papers in these areas. 